\title{
Use of functional magnetic resonance imaging in patients with irritable bowel syndrome and functional dyspepsia
}

\author{
Katarzyna Skrobisz¹, Grażyna Piotrowicz², Adrianna Drozdowska ${ }^{3}$, Karolina Markiet ${ }^{3}$, Agnieszka Sabisz $^{3}$, \\ Patrycja Naumczyk ${ }^{4}$, Grażyna Rydzewska ${ }^{5,6}$, Edyta Szurowska ${ }^{3}$ \\ ${ }^{1}$ Department of Radiology, Medical University of Gdansk, Gdansk, Poland \\ ${ }^{2}$ Department of Gastroenterology, Self-Dependent Health Care Unit of Ministry of Interior, Gdansk, Poland \\ ${ }^{3} 2^{\text {nd }}$ Department of Radiology, Medical University of Gdansk, Gdansk, Poland \\ ${ }^{4}$ Institute of Psychology, University of Gdansk, Gdansk, Poland \\ ${ }^{5}$ Department of Internal Medicine and Gastroenterology with IBD Subdivision, Central Clinical Hospital of the Ministry \\ of the Interior, Warsaw, Poland \\ ${ }^{6}$ Faculty of Health Sciences, Jan Kochanowski University, Kielce, Poland
}

Gastroenterology Rev 2019; 14 (3): 163-167

DOI: https://doi.org/10.5114/pg.2019.88163

Key words: irritable bowel syndrome, functional dyspepsia, functional magnetic resonance imaging, resting state.

Address for correspondence: Grażyna Piotrowicz MD, PhD, Department of Gastroenterology, Self-Dependent Health Care Unit of Ministry of Interior, 4/6 Kartuska St, 80-104 Gdansk, Poland, phone: +48 602784 507, e-mail: piotrowicz.grazyna@interia.eu

\begin{abstract}
Functional brain imaging (positron emission tomography - PET, functional magnetic resonance imaging - fMRI), allowing in vivo analysis of the brain-digestive tract interaction and the neurological mechanisms underlying visceral hypersensitivity, significantly advanced research and helped in the understanding of the interrelations in this field. Differences in this parameter can result from alterations in task-related cognitive states or from resting state processes. Nowadays, advanced imaging techniques such as fMRI are more frequently used and are acknowledged among both clinicians and radiologists in the diagnostic algorithm of digestive tract diseases. Functional dyspepsia is a condition in which neuroimaging allows for analysis of dysfunctions within the brain-gut axis (BGA) engaged in processing of visceral discomfort and pain. The results of studies in patient groups with irritable bowel syndrome prove that psychosocial factors significantly affect the mechanisms regulating visceral sensitivity within the brain. The BGA includes neuronal pathways (autonomic nervous system), neuroendocrine (hypothalamo-pituitary-adrenal axis), and neuroimmunological ones. Psychological processes affect the functioning of the digestive system and can cause dyspeptic symptoms. A patient's mental condition associated with stress can affect processes taking place in the central nervous system and trigger somatic reactions in the digestive tract through the autonomic visceral system.
\end{abstract}

\section{Introduction}

Functional magnetic resonance imaging ( $\mathrm{fMRI})$ is one of the imaging methods designed to assess regional changes in oxygenation and blood flow within the brain. An increase in the active region volume is measured with BOLD (blood-oxygenation-level-dependent), which specifies the relation between magnetic resonance imaging (MRI) signal intensity and blood oxygenation level, with image intensity changing depending on deoxyhaemoglobin level. Differences in this parameter can result from alterations in task-related cognitive states or from resting state processes.
In 1982 Keith Thulborn investigated differences in magnetic dependence of oxy- and deoxyhaemoglobin with MRI in order to measure the oxygen consumption by the brain. His studies showed that it is possible to measure blood oxygenation in vitro with MRI, which was a significant step in developing the combination of fMRI and BOLD $[1,2]$.

In the 1990s, fMRI started to be more commonly used in neurological studies mainly devoted to the evaluation of the cognitive functions of the brain, in psychiatry, in clinical psychology, and in preventive planning. The popularity of FMRI nowadays results from the wide 
availability of the devices (1.5 T scanners), non-invasiveness of the method, and good spatial resolution. The method is more and more frequently used as a biomarker for numerous illnesses, in therapy monitoring, neurological surgery planning, and in investigating pharmacological efficacy.

BOLD, as previously mentioned, is the most commonly used fMRI technique. It involves detection of deoxygenated haemoglobin in blood because it presents with paramagnetic properties, as well as identification of changes in its regional distribution dependent on neuronal activation. $\mathrm{fMRI}$ in resting state is a relatively new tool, allowing the analysis of connections between functionally connected but anatomically separated brain regions with no need to perform any particular active tasks.

It is commonly known, based on daily observation and scientific research, that psychological processes, both affective and cognitive, affect the functioning of the digestive system. The relation between psycholog ical factors and visceral hypersensitivity has been implied for a long time, mainly based on studies focused on functional diseases of the digestive system. However, until quite recently, the mechanism of this remained unclear, mainly due to a lack of non-invasive investigation methods. Functional brain imaging (positron emission tomography - PET, fMRI), allowing in vivo analysis of the brain-digestive tract interaction and neurological mechanisms underlying visceral hypersensitivity, significantly advanced research and helped in understanding the interrelations in this field.

The brain-gut axis (BGA) is a notion introduced in the course of research, which can be defined as a bidirectional system of connections between the brain and the digestive tract, including not only neuronal pathways (autonomic nervous system) but also neuroendocrine (hypothalamo-pituitary-adrenal axis) and neuroimmunological ones. There are two independent systems transferring signals from the intestines to the brain: the parasympathetic and the sympathetic afferent system. The parasympathetic afferent neurones project to the solitary nucleus and then through the thalamus (periventricular nucleus) to the hypothalamus, locus coeruleus-amygdala circuit and the periaqueductal grey, i.e. brain structures engaged in emotional autonomic, neuroendocrine, and behavioural response [3]. One third of all neurons originating from the thalamus transfer signals from the intestine to the visceral sensory neuromatrix [4, 5]. The sympathetic afferent neurones create medullary-lentiform pathways, medullary-intracerebral, and medullary-hypothalamic pathways responsible for automatic reflexes to visceral stimulation. The key pathway is the spinothalamic pathway projecting to the somatosensory cortex (SI/SII), callosal gyrus cortex, and the insula, where the conscious visceral reflex is produced. The main function of SI/SII is to code the intensity and location of visceral stimuli, while the callosal gyrus cortex is mainly engaged in affective-motivative aspects (anxiety-related pain) and cognitive-assessment aspects of pain (prediction). Within the insula sensory information of various senses is processed, pertaining to the inner condition of the body. It is not a pain-specific region, but it is engaged in coding sensory and emotional aspects of pain, by integrating visceral and somatic sensory stimuli with emotional information. The highest structure integrating and processing sensory and visceral pain signals is the prefrontal cortex, which is also engaged in forming responses to sensory stimuli [6].

The classification of functional disorders of the digestive tract is based on the Rome Criteria published in 2006. The most common complaint belonging to the group of functional disorders is functional dyspepsia (FD), occurring in approximately $25 \%$ of the general population. The prevalence of irritable bowel syndrome (IBS) in western countries is estimated at 15-24\%. Complaints associated with functional conditions are more common in women than in men, with an estimated women:men ratio of $2-3: 1$, and they are more frequent in the young population [7].

Studies conducted on healthy participants provide significant and direct proof confirming that there is extensive modulation of normal sensory signals coming from the intestines by the psychological processes, both affective and cognitive, at the brain level. Even though this does not necessarily indicate that the symptoms observed in patients with functional diseases of the digestive tract are directly caused by these processes, a hypothesis has been formed that they may play a role at least in some of these patients [8-10].

\section{Irritable bowel syndrome (IBS)}

It has been shown that during rectal distension in healthy participants the same regions of brain (neuromatrix) are activated as in the case of patients with IBS. However, the intensity and the range of activation is different in IBS groups vs. healthy control groups, mainly in regions engaged in processing affective and cognitive aspects of visceral sensitivity/pain. In some studies, the anterior cingulate cortex-anterior mid cingulate cortex (ACC-aMCC) activity during painful rectal distension was higher in FD patients, compared to healthy participants [11-14]. These differences can result from hyperregulation of the afferent visceral stimuli to the brain, various affective and cognitive reactions at the brain level (increased prediction and vigilance), or negative 
affective reaction to sensory visceral stimuli. However, it should be pointed out that in many studies involving rectal distension in patients with IBS, results indicated decreased ACC-aMCC activity or no such activity at all [15-20]. These observations can be associated with poor descending antinociceptive response through the pathways coming from the ACC level, the "ceiling effect", or the differentiating sensitivity of the lateral and medial system of pain in IBS patients. Contradictory observations were also made in studies on the response to somatic pain stimuli in IBS patients, which showed both lower [20], similar [21, 22], and higher [21] somatic pain thresholds, compared to healthy participants. Based on fMRI scans, Ringel et al. [23] observed that IBS patients with abuse history presented with higher activity of MCC and posterior callosal gyrus during attempts of rectal distension, compared to IBS patients with no such history [23]. On the other hand, ACC activity (perigenual ACC) in this group was lower compared to IBS patients without abuse history, and with healthy participants. However, it is earlier studies with the use of PET showed lower ACC-aMCC (ACC) activity during rectal distension attempts in patients with IBS [15]. Despite the discrepancies, the results of the studies prove that psychosocial factors significantly affect the mechanisms regulating visceral sensitivity within the brain. Lawal et al. [24] conducted a study with the use of fMRI to assess regional brain activation during subliminal complaints evoked by rectal distension (below the threshold of conscious perception). The total volume of the activated (simulated) cortex was significantly higher in patients with IBS compared to the control group. As reported by researchers, use of subliminal stimuli allowed them to analyse the processing of afferent visceral signals by the brain, independently of the cognitive modulation. With this method they showed that visceral hypersensitivity in patients with IBS is probably caused by increased afferent signalling/stimulation to the brain, rather than different processing of signals at the brain level.

\section{Functional dyspepsia (FD)}

Functional dyspepsia is another condition in which neuroimaging allows the analysis of dysfunctions within the brain-gut axis engaged in processing of visceral discomfort and pain. Patients with FD present with various abnormalities in some specific brain areas. Vandenberghe et al. examined FD patients with the use of gastric balloons and observed abnormal activity within the precentral gyrus (bilaterally), frontal inferior gyrus (bilaterally), frontal central gyrus, temporal superior gyrus, both cerebellar hemispheres, and the left temporal inferior gyrus [25]. Van Oudenhove et al. showed that abnormal brain stimulation observed in patients with FD and in healthy people, in various experimental circumstances (gastric distension, no distension, sham distension), was associated with sensory, agitation-anxiety, and affective-cognitive disturbances [26].

Researchers also investigated disturbances that occur in resting state in patients with FD. Van Oudenhove et al. showed that psychosocial factors can contribute to abnormal brain activity in patients with FD in resting state. Their studies showed that the activity of pACC (pregenual anterior cingulate cortex) and aMCC (anterior middle circulate cortex) was negatively correlated with anxiety, while activity of the dorsal part of the pons showed positive correlation. Moreover, they proved that abuse history was associated with different activity within the insula, prefrontal cortex (PFC), hippocampus, and the corpus callosum $[26,27]$. On the other hand, Liu et al. [28] carried out a study with 30 FD patients and 30 healthy participants (control group) and observed that differences in activity in these groups included the right dorsal-medial prefrontal cortex, left ventral-medial prefrontal cortex, left orbital-frontal cortex, right supplementary motor area (SMA; secondary motor cortex, frontal lobe), right temporal pole, insula, right callosal gyrus, left thalamus, hippocampus, parahippocampal gyrus, and the right cerebellum. In another study [29] the same team of researchers examined 49 patients and 39 healthy participants (control group) to find significant differences in the activity within the dorsal-medial prefrontal cortex, left ventral-medial prefrontal cortex, orbital-frontal cortex, anterior part of the callosal gyrus, thalamus, parahippocampal gyrus, precuneus, parietal cortex, and temporal pole. Moreover, changes in the default mode network (DMN) were consistent with symptom progression in the course of FD. A different study conducted by Zhou et al. [30] analysed interhemispheric resting-state functional connectivity in 26 patients with FD and 20 healthy participants. They showed that patients with FD have more interhemispheric functional connections, both general and region-specific, such as within anterior callosal gyrus, insula, and/or the thalamus, compared to the healthy population.

\section{Discussion and conclusions}

Functional brain imaging is a valuable method in investigating neural mechanisms underlying visceral disturbances. Dyspeptic complaints do not correlate well with any particular physiological disturbances. However, a correlation was found between the signs of early satiation and disorders accompanying gastritis and duodenitis (mainly eosinophilia). There are also genetic studies conducted into possible correla- 
tion between bacterial inflammation of the upper and lower segments of the digestive tract and functional disorders.

The most recent studies show that complex biological interactions and behavioural mechanisms cause the complaints in functional disorders in both the lower and upper segment of the digestive tract. A patient's mental condition associated with stress can affect the biological processes taking place in the central nervous system and trigger somatic reactions in the digestive tract through the autonomic visceral system. Studies suggest that dyspeptic symptoms can be caused by psychological factors. There was a correlation observed between anxiety and decreased pain threshold, epigastric discomfort, burning sensation, and early satiety. Patients with functional disorders of the digestive tract are also less effective in dealing with daily-life problems. They are more inclined to be too critical of themselves, have catastrophic thoughts, or focus on their failures. There was no relation observed between complaints associated with functional disorders and survival [30-32].

There have been numerous studies carried out to investigate the neuroanatomy of emotions and to identify brain structures activated during an experiment based involving evoking various emotional states in healthy people. Phan et al. carried out a meta-analysis of 55 studies involving PET and $\mathrm{FMRI}$ examinations, which indicated that the feeling of fear is associated with increased activity of the amygdalae, and sadness with activation of the subgenual part of the callosal gyrus.

Overlapping of various functional disorders questions the differences amongst them, which is consistent with the general trend of perceiving functional disorders as a mask of other diseases and their possible psychological/emotional background.

\section{Conflict of interest}

The authors declare no conflict of interest.

\section{References}

1. Ogawa S, Sung Y. Functional magnetic resonance imaging Scholarpedia 2007; 2: 3105.

2. Raichle M. A brief history of human brain mapping. Trends Neurosci 2009; 32: 118-26.

3. Dunckley P, Wise RG, Fairhurst M, et al. A comparison of visceral and somatic pain processing in the human brainstem using functional magnetic resonance imaging. J Neurosci 2005; 25: 7333-41.

4. Van Oudenhove L, Demyttenaere K, Tack J, Aziz Q. Central nervous system involvement in functional gastrointestinal disorders. Best Pract Res Clin Gastroenterol 2004; 18: 663-80.

5. Jones MP, Dilley JB, Drossman D, Crowell MD. Brain-gut connections in functional Gl disorders: anatomic and physiologic relationships. Neurogastroenterol Motil 2006; 18: 91-103.
6. Price DD. Psychological and neural mechanisms of the affective dimension of pain. Science 2000; 288: 1769-72.

7. Olafsdottir LB, Gudjonsson H, Jonsdottir HH, Thjodleifsson B. Stability of the irritable bowel syndrome and subgroups as measured by three diagnostic criteria - a 10-year follow-up study. Aliment Pharmacol Ther 2010; 32: 670-80.

8. Phillips ML, Gregory LJ, Cullen S, et al. The effect of negative emotional context on neural and behavioural responses to oesophageal stimulation. Brain 2003; 126: 669-84.

9. Gregory LJ, Yágüez L, Williams SC, et al. Cognitive modulation of the cerebral processing of human oesophageal sensation using functional magnetic resonance imaging. Gut 2003; 52: 1671-7.

10. Yágüez L, Coen S, Gregory LJ, et al. Brain responseto visceral aversive conditioning: a functional magnetic resonance imaging study. Gastroenterology 2005; 128: 1819-29.

11. Mertz H, Morgan V, Tanner G, et al. Regional cerebral activation in irritable bowel syndrome and control subjects with painful and nonpainful rectal distention. Gastroenterology 2000; 118 : 842-8.

12. Naliboff BD, Derbyshire SW, Munakata J, et al. Cerebral activation in patients with irritable bowel syndrome and control subjects during rectosigmoid stimulation. Psychosom Med 2001; 63: 365-75.

13. Mayer EA, Berman S, Suyenobu B, et al. Differences in brain responses to visceral pain between patients with irritable bowel syndrome and ulcerative colitis. Pain 2005; 115: 398-409.

14. Verne GN, Himes NC, Robinson ME, et al. Central representation of visceral and cutaneous hypersensitivity in the irritable bowel syndrome. Pain 2003; 103: 99-110.

15. Ringel Y, Drossman DA, Turkington TG, et al. Regional brain activation in response to rectal distension in patients with irritable bowel syndrome and the effect of a history of abuse. Dig Dis Sci 2003; 48: 1774-81.

16. Wilder-Smith $\mathrm{CH}$, Schindler D, Lovblad K, et al. Brain functional magnetic resonance imaging of rectal pain and activation of endogenous inhibitory mechanisms in irritable bowel syndrome patient subgroups and healthy controls. Gut 2004; 53: 1595-601.

17. Andresen V, Bach DR, Poellinger A, et al. Brain activation responses to subliminal or supraliminal rectal stimuli and to auditory stimuli in irritable bowel syndrome. Neurogastroenterol Motil 2005; 17: 827-37.

18. Kwan CL, Diamant NE, Pope G, et al. Abnormal forebrain activity in functional bowel disorder patients with chronic pain. Neurology 2005; 65: 1268-77.

19. Bernstein CN, Frankenstein UN, Rawsthorne P, et al. Cortical mapping of visceral pain in patients with GI disorders using functional magnetic resonance imaging. Am J Gastroenterol 2002; 97: 319-27.

20. Verne GN, Robinson ME, Price DD. Hypersensitivity to visceral and cutaneous pain in the irritable bowel syndrome. Pain 2001; 93: 7-14.

21. Chang L, Mayer EA, Johnson T, et al. Differences in somatic perception in female patients with irritable bowel syndrome with and without fibromyalgia. Pain 2000; 84: 297-307.

22. Whitehead WE, Holtkotter B, Enck P, et al. Tolerance for rectosigmoid distention in irritable bowel syndrome. Gastroenterology 1990; 98: 1187-92. 
23. Ringel Y, Drossman DA, Leserman JN, et al. Association between central activation and pain reports in women with IBS. Gastroenterology 2006; 130: A77-8.

24. Lawal A, Kern M, Sidhu $\mathrm{H}$, et al. Novel evidence for hypersensitivity of visceral sensory neural circuitry in irritable bowel syndrome patients. Gastroenterology 2006; 130: 26-33.

25. Vandenberghe J, Dupont P, Van Oudenhove L, et al. Regional cerebral blood flow during gastric balloon distention in functional dyspepsia. Gastroenterology 2007; 132: 1684-93.

26. Van Oudenhove L, Vandenberghe J, Dupont P, et al. Abnormal regional brain activity during rest and (anticipated) gastric distension in functional dyspepsia and the role of anxiety: a $\mathrm{H}(2)$ (15)O-PET study. Am J Gastroenterol 2010; 105: 913-24.

27. Van Oudenhove L, Vandenberghe J, Dupont P, et al. Regional brain activity in functional dyspepsia: a H(2)(15)O-PET study on the role of gastric sensitivity and abuse history. Gastroenterology 2010; 139: 36-47.

28. Liu P, Qin W, Wang J, et al. Identifying neural patterns of functional dyspepsia using multivariate pattern analysis: a resting-state FMRI study. PLoS One 2013; 8: e68205.

29. Liu P, Zeng F, Zhou G, et al. Alterations of the default mode network in functional dyspepsia patients: a resting-state fmri study. Neurogastroenterol Motil 2013; 25: e382-8.

30. Zhou G, Liu P, Zeng F, et al. Increased interhemispheric resting-state functional connectivity in functional dyspepsia: a pilot study. NMR Biomed 2013; 26: 410-5.

31. Olafsdottir LB, Gudjonsson H, Jonsdottir HH, Thjodleifsson B. Stability of the irritable bowel syndrome and subgroups as measured by three diagnostic criteria - a 10-year follow-up study. Aliment Pharmacol Ther 2010; 32: 670-80.

32. Chang JY, Locke GR 3rd, McNally MA, et al. Impact of functional gastrointestinal disorders on survival in the community. Am J Gastroenterol 2010; 105: 822-32.

Received: 13.03.2019

Accepted: 14.05 .2019 